\title{
Radiologic appearance of stress fractures
}

\author{
DANIEL ARROWSMITH, DO
}

The large numbers of Americans involved in sports and physical activities have made stress fractures a more common injury to the lower extremities than before. Presented are the typical radiographic and scintigraphic appearances of stress fractures and the appropriate medical imaging with which to diagnose this entity.

It has been estimated ${ }^{1}$ that $38 \%$ of all Americans are involved in a fitness program, and, the number of joggers has been projected ${ }^{2}$ at 37.2 million. As a result of this interest in vigorous activity, stress fractures are becoming increasingly common occurrences, and the primary care physician should be aware of their symptoms and methods of diagnosis. The purposes of this article are to demonstrate some of the more common stress fractures and to discuss the medical imaging necessary to establish their diagnosis.

\section{Case history}

Figure 1 demonstrates radiographic and scintigraphic studies in an 18-year-old man. The patient recently had begun a vigorous jogging program. Three weeks before the nuclear medicine scan of Sept 5, 1984, he consulted his physician for pain in both proximal tibial regions.

At the time this paper was written, Dr Arrowsmith was radiologist at the New Valley Osteopathic Hospital, Yakima, Wash. He is currently associate radiologist at Metropolitan Hospital, Pinellas Park, Fla.

Reprint requests to Daniel Arrowsmith, DO, Radiology Department, Metropolitan Hospital, 7950 66th St N, Pinellas Park, FL 33546.
The onset of pain had been gradual, and he could not recall a specific traumatic incident. The radiographs taken at that initial visit were normal (not illustrated). The patient was treated symptomatically, but the pain persisted. The September scintiscans (Fig 1) showed increased radionuclide activity in the proximal tibial region bilaterally. While not a specific finding, the diagnosis of bilateral stress fractures was highly suggested. Radiographs subsequently taken 1 week later revealed linear areas of sclerosis in the proximal tibias compatible with stress fractures.

\section{Discussion}

Stress fractures are mechanical disturbances of trabeculas that occur in normal bone subjected to repeated episodes of minor stress. In contrast to traumatic fractures, which occur abruptly at the time of injury and are associated with a loss of structural continuity, stress fractures develop during a period of days and weeks. In the early stages, stress fractures are not visible, nor do they show displacement or change in bone alignment. ${ }^{1}$

Stress fractures were initially and primarily described in the early 1900 s by military physicians. The first case reported by physicians in private practice was in 1940 , by Weaver and Francisco. ${ }^{2}$ The authors proposed the term pseudofracture to describe a lesion that always occurred in the upper third of one or both tibias and was characterized on roentgenograms by a localized area of periosteal thickening and new bone formation over what appeared to be an incomplete V-shaped fracture in the cortex. They incorrectly concluded that these cases were the result of a nonsup- 

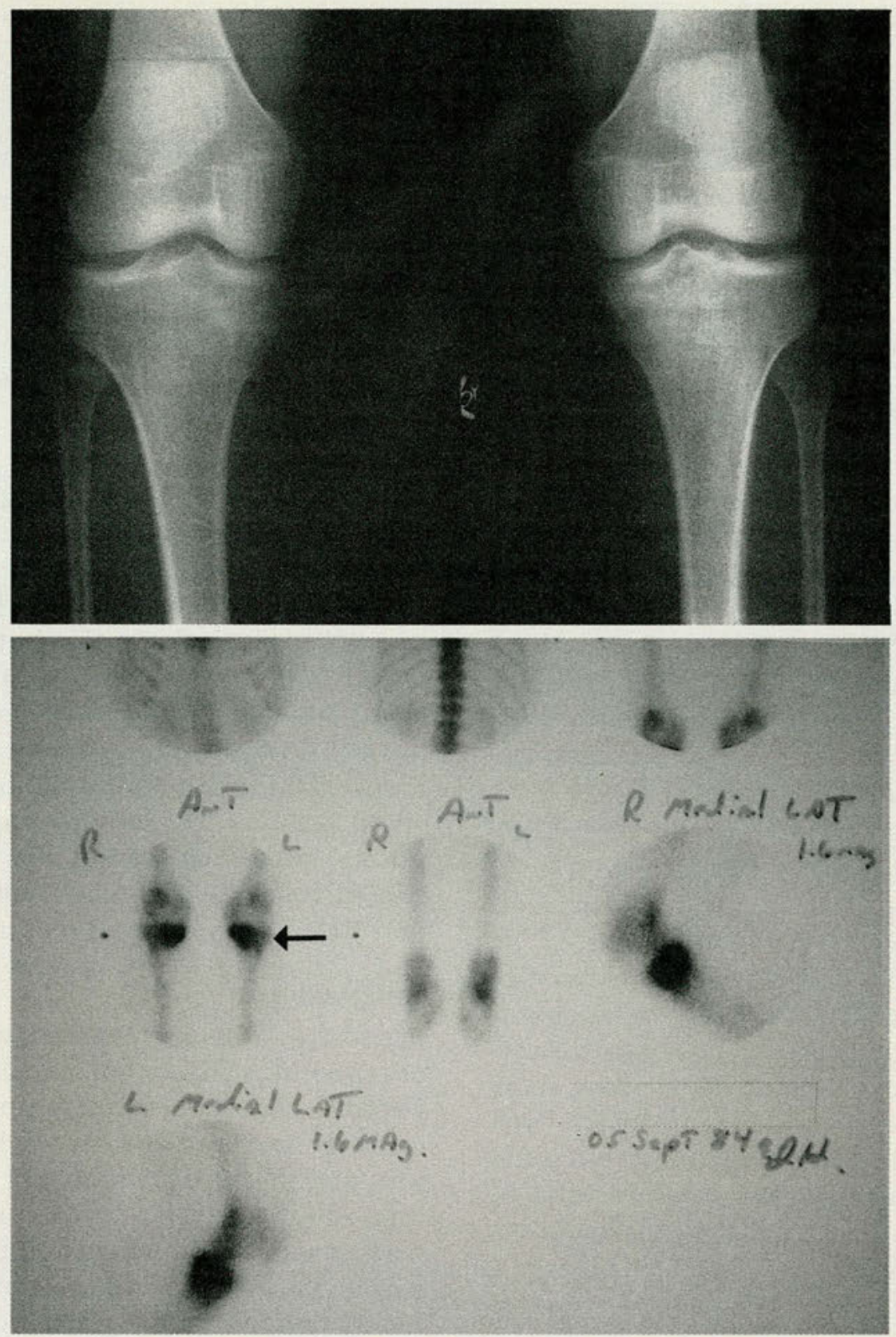

Figure 1. Bilateral proximal tibial stress fractures appear in radiograph (top) and in nuclear medicine bone scan (bottom). Increased radionuclide activity is indicative of stress fractures (arrow).

purative osteomyelitis from which causative organisms were never isolated.

Many synonyms for stress fracture have been used, including pseudofracture, spontaneous fracture, fatigue fracture, march fracture, Deutschlander's fracture, and exhaustion fracture. Devas ${ }^{3}$ has written extensively on stress fractures and their radiologic characteristics. He has emphasized the marked delay in the radiographic appearance of stress frac- tures. This delay may be as long as 3 weeks. Consequently, the diagnosis is based on historical and physical findings and is often confirmed by nuclear medicine bone scan with radiography.

In general, stress fractures occur in military recruits and in athletes, especially those who recently have begun an exercise program or those who have abruptly intensified their exercise regimen. The physician who examines 
such a patient should be aware of the possibility of this clinical entity.

At the molecular level, the normal response to repeated stress is a remodeling process. ${ }^{4}$ The initial stage is bone resorption, followed by the formation of new bone in a pattern more appropriate for the new stress. Because new bone formation is significantly slower than the original process, the bone weakens. With continued stress, the weakening can result in a fracture, usually of a hairline type. Bone does not gain strength as rapidly as muscle, which may explain why most of the runners who maintain a level of training are not potential candidates for a stress fracture.

The diagnosis of stress fracture is based on a careful history, thorough physical examination, and appropriate medical imaging studies. The physician must maintain a high index of suspicion when a patient complains of pain of insidious onset, usually noticed at the end of a workout, and not related to a specific event. The pain increases in intensity with further exercise and is relieved with rest. Often there is a history of a recent change in the exercise pattern. For example, some patients might increase their distance or speed; others might run on a harder than usual surface or with shoe soles that are thinner than before.

Radiographs and nuclear medicine bone scintiscans should be obtained in patients with a history of recently increased physical activity and physical examination findings compatible with possible stress fracture. Radiography is the usual initial procedure. If these are normal and the clinical impression of stress fracture remains, bone scintigraphy with emphasis on the symptomatic area are then performed. The bone scan is often abnormal, sometimes in several areas, some of which are clinically less symptomatic but still painful. Additional radiographs obtained later, at intervals of up to 1 month or longer, are often helpful in certain cases in which the initial radiographs are normal or equivocal.

Stress fractures can involve several bones in one or both lower extremities, including femoral necks, pelvis, tibia (Fig 2), and fibulas. The fractures also quite commonly involve the os calcis and metatarsal areas (Fig 3), and are often bilateral. Wilson and $\mathrm{Katz}^{5}$ reported cal-

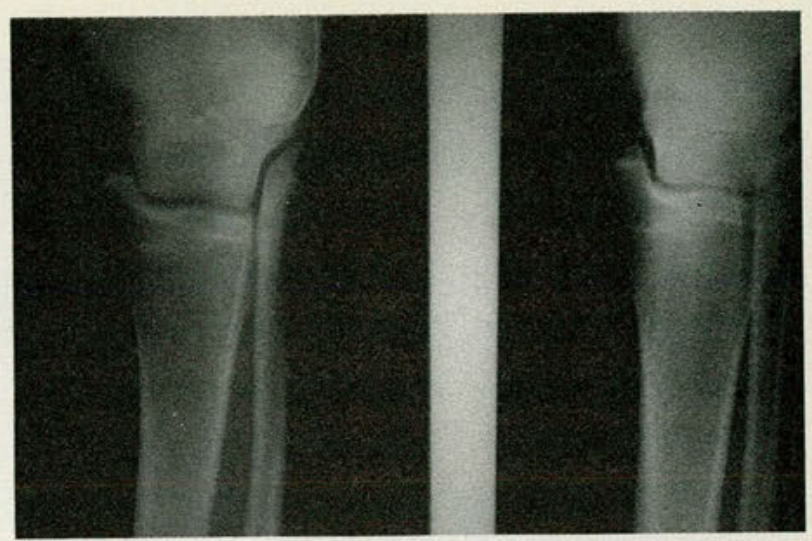

Figure 2. Distal tibial stress fracture.

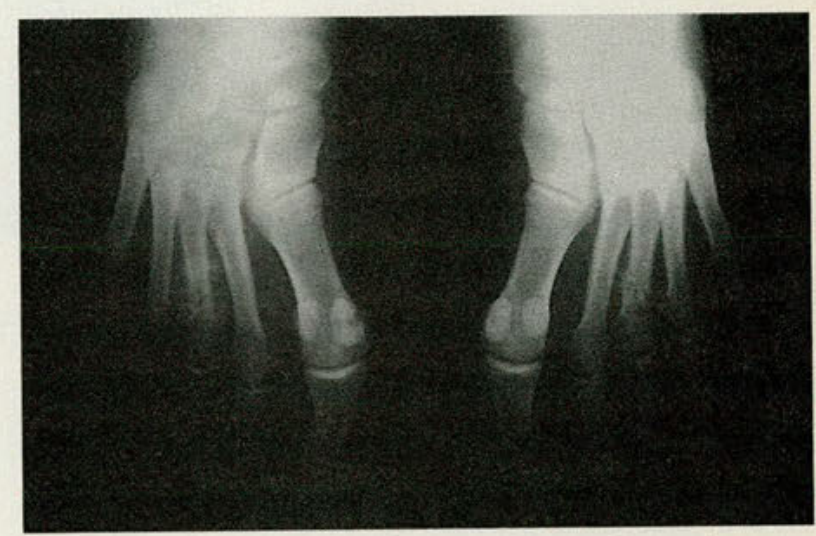

Figure 3. Bilateral metatarsal stress fractures.

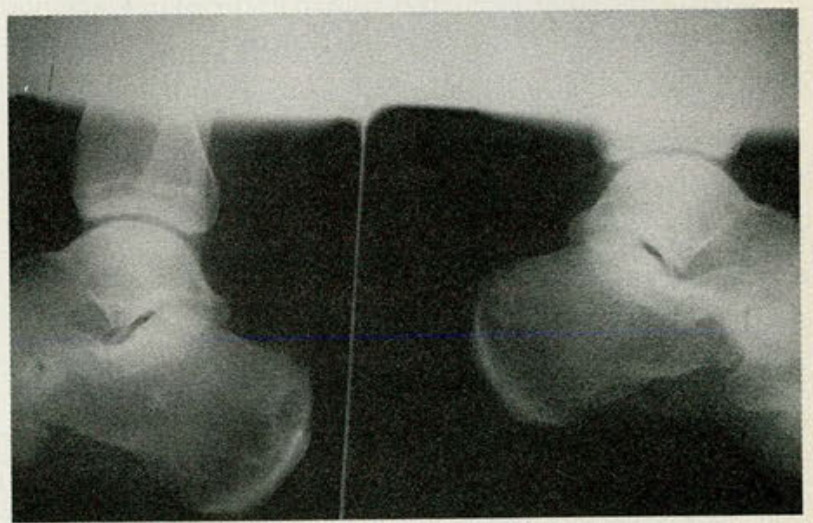

Figure 4. Bilateral calcaneal stress fractures.

caneal stress fracture (Fig 4) to be bilateral in $27 \%$ of the cases in their series.

Stress fracture may not be visible radiographically for as long as 4 weeks after the onset of symptoms. ${ }^{6}$ Before this time, the radiographs may be normal. The first visible sign may be subperiosteal new bone, which appears as a hazy area of thin callus (Fig 5). The area of the fracture is characterized by a sclerotic 


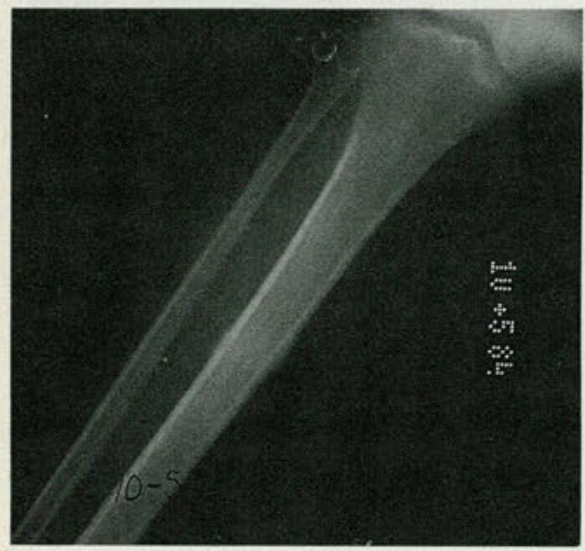

Figure 5. Left: Radiograph demonstrates stress fracture. Right: Bone scan shows multiple sites of increased uptake (arrows)indicating stress fractures not visible radiographically.

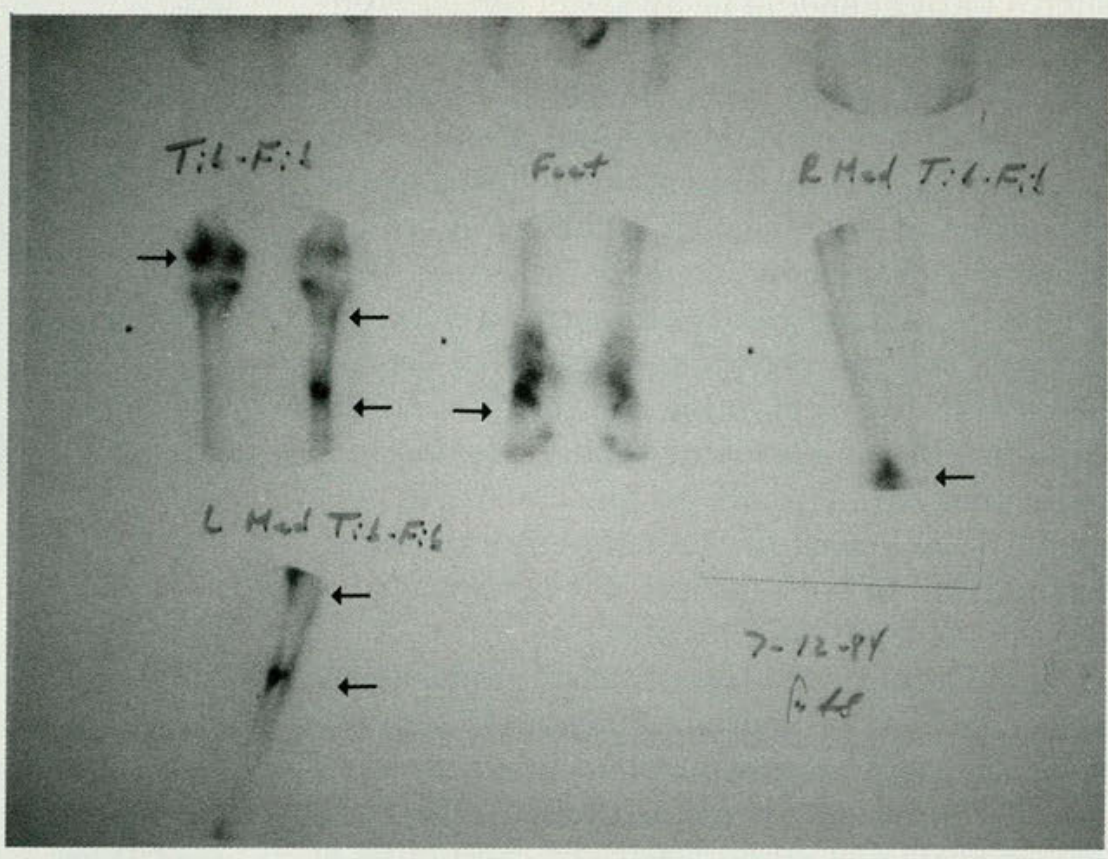

band or consolidation of callus along the fracture line. There also may be diffuse thickening of the cortex and subsequent narrowing of the medullary canal. ${ }^{7}$ Often a definite radiolucent fracture will not be seen. Stress fracture of the fibula and metatarsals may be initially visualized by periosteal reaction only, with callus developing later (Fig 6).

Scintigraphy is quite useful to detect stress fracture before it can be seen radiographically. The scan shows increased radionuclide activity in the suspected region of the fracture. When a stress fracture has been verified radiographically, other areas of increased radionuclide uptake also should be regarded as probable fracture sites, even if none is radiographically visualized. Thus, stress fracture is often considered a multifocal entity.

\section{Summary}

In conclusion, the primary care physician or other physicians who see patients who are active in sports should be aware of stress fractures. Radiography can be quite helpful in the detection of the abnormality, but in the absence of radiographic findings, nuclear medicine bone scintigraphy should be considered an early and accurate means for the diagnosis.

1. Murray RO, Jacobson HG: Radiology of Skeletal Disorders,

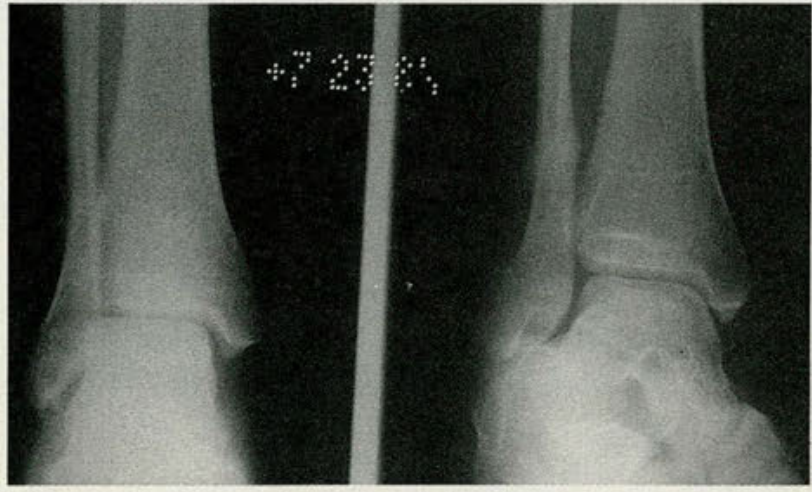

Figure 6. Stress fracture of the distal fibula.

Exercises in Diagnosis. Baltimore, Williams \& Wilkins, 1971, p 130.

2. Weaver JB, Francisco CB: Pseudofractures. J Bone Joint Surg 1940;22:610-615.

3. Devas M: Stress Fractures. New York, Churchill \& Livingston, 1975, pp 1, 2, 19-27, 113-116.

4. Dugan RC, D'Ambrosia R: Fibular stress fractures in runners. J Family Practice 1983;17(3)415-418.

5. Wilson ES Jr, Katz FN: Stress fractues: An analysis of 250 consecutive cases. Radiology 1969;92:481-486.

6. Devas MB, Sweetnam R: Stress fractures of the fibula. JBone Joint Surg 1956;38:818-829.

7. Murray R, Jacobsen H: The Radiology of Skeletal Disorders. Baltimore, Williams \& Wilkins, 1971.

Greaney RB, Gerber FH, Laughlin RL, et al: Distribution and natural history of stress fractures in U.S. Marine recruits. $R a$ diology 1983;146:339-346.

Roub LW, Gumerman LW, Hanley EN, et al: Bone stress: A radionuclide imaging perspective. Radiology 1979;132:431438.

Rupani HD, Holder LE, Espinola DA, et al: Three-phase radionuclide bone imaging in sports medicine. Radiology 1985;156:187-196. 